\title{
Research of Firewall Technology
}

\author{
Meilin $\mathrm{Wu}^{1, \mathrm{a}}$ and Sen $\mathrm{Li}^{1, \mathrm{~b} *}$ \\ ${ }^{1}$ Xinjiang Shihezi Vocational Technical College, Xinjiang, China \\ a59685686@qq.com, b22203719@qq.com
}

\begin{abstract}
Keywords: Network security; Firewall technology; Information security; Security technology; Computer net
\end{abstract}

\begin{abstract}
Through the firewall can define one of the key points to prevent invasive; monitoring network security and give the alarm in case of abnormalities, especially for the great amount of information through the removal of inspection, but also should make log register; provides network address conversion function, help to alleviate the IP address resource issues. So use a firewall to protect computer, server and a local area network, which to a certain extent avoid attack and protect the safety of cyber source is a current popular technologies and means.
\end{abstract}

\section{Introduction}

The application of computer technology and development and promote the information technology revolution[1], computer and information technology and its extensive penetration and affinity, in aspects affect the world economy and social development[2]. In twenty-first Century, the human life and the Internet more closely[3], more closely related to the. However, various problems have appeared, such as the Internet, there are many threats and attacks, the network security is damaged, the light leakage of information[4], the system platform and the cyber source being attacked. Therefore, how to ensure the information is true, complete, effectively, legally transceiver, the problem of network security has become a hotspot[5]. But the problems of network security are the most common method is to take the firewall technology[6].

In general, a firewall is a system or a set of system, in the internal network and external network to perform certain security strategy[7]; it is actually a kind of isolation technology. An effective firewall should be able to ensure that all from the Internet into or to the Internet information will pass through the firewall[8], all through the firewall information should be inspected. Through the firewall can define one of the key points to prevent invasive; monitoring network security and give the alarm in case of abnormalities, especially for the great amount of information through the removal of inspection, but also should make log register[9]; provides network address conversion function, help to alleviate the IP address resource issues[10]. So use a firewall to protect computer, server and a local area network, which to a certain extent avoid attack and protect the safety of cyber source is a current popular technologies and means[11].

\section{The Concept and Functions of the Firewall}

The firewall is refers to the isolation between external network and local network of a network control system[12]. Its filtering of network data transmission between has a certain role, to shield the internal information structure and running condition to provide security and audit control, thus reach the purpose of protection of internal information. The essence of a firewall is a kind of separation technology; its core idea is to build relative internal network environment, and its logical separation. Firewall's main function is to control the unsafe service, filtering illegal user, make the network inside from outside attacks; Special site to limit the access to the network, blocking access to certain host; Centralized security protection, simplify network management, network Settings password or other proof of identity on the firewall is more superior to access the host; Firewall through the blockade of domain name service, prevent the network attack to obtain useful information; Through the study of the statistical analysis of passes through the firewall access, allowing them to better use of network resources[13]; Organizations may, according to the special 
requirements of this unit configuration firewall system, implement safety control.

\section{The Firewall Technology Classification}

Packet Filtering Technology. Packet filter module work mainly focused on network layer, it can accurately on the traditional link layer by checking each code source address, transmission protocol and port information matching with preset security policy[14], to decide whether or not a message through, according to the serial number and handshake sequence logic analysis for effective judgment, can be more effective against related to virus attacks. Packet filtering technology to standard routing software are built in it, no additional cost, is transparent to users and application at the same time, don't need the user login and password, run faster. But configuration access control list in early is more complex, need to be well informed about network management for network services. Meanwhile packet filtering technology lack the ability to track, not from computer hackers found in log records, simply check the address and port, the application of the link layer protocol can't prevent, can't understand the context of a specific service environment and data[15].

Proxy Service Technology. Agent service layer is a special kind of application code is in the network to allow or deny specific applications and services, implementation of data traffic monitoring, filtering and reporting capabilities. Agent working principle is simple, the user simply with the proxy server connection is established, and then inform destination agent, to conform to the request, the agent with their own identity and purpose site connection is established, then the agent forward data in these two connections[16]. Agent service technology can effectively achieve the state and part of the transmission on network application of effective information, to provide comprehensive audit and log function, for each specific application has a corresponding agent module, to ensure the effect of the firewall security. Proxy firewall's most prominent advantages for safety, namely every inside and outside the network needs a specific service access, submit a request and response, by the firewall itself does not give computer any chance of the session, the internal and external network security is extremely high. But the agent technology is relatively slow, the user of the internal and external network gateway network problem is easy to appear when a higher throughput requirements, some agents need to modify the client software, has brought great inconvenience to the customer.

The Condition Monitoring Technology. Condition monitoring technology is also called dynamic packet filtering, is an extension of the traditional packet filter, monitoring module supports a variety of protocols and applications, realize effective expansion of application, it can effectively overcome the firewall technology limited, high security, ensure all the original information through the network can effectively monitor, effectively improve the safety range[17]. Packets in the low-level processing and block, saves execution time, improve the execution efficiency. Established after the firewall, the system will no longer need to deal with this link, condition monitoring also has the advantage that the scalability and extensibility, widely used in the monitoring system. But in the test and analysis, technology monitoring network connection will be caused by some kind of hysteresis, especially in the case of multiple connection activated at the same time, there will be a caton phenomenon.

\section{The Firewall Technology of Network Application}

Internal Web Applications. Unit of the Intranet in general is to ban all Internet users to access, if there is a need to connect to the Internet, the allowed permission is very low, with a real server data is within the protected network host. Through the network address translation technology will host the protected internal network address mapping in effective gateway firewall, can not only external mask internal network structure and common gateway address, to protect the safety of internal network, but also can greatly save the cost of investment[18]. By setting a packet filter firewall server port, only open for the function of the internal network, by analyzing the packet data packet filtering. Therefore, often used in the Intranet processor packet filter firewall technology, the firewall is transparent to users, legitimate users into the network, don't feel the existence of the 
firewall, use rise very convenient.

The Application of Boundary Router. Proxy firewall technology is often applied to the border router to use. Before units existing border router, can make full use of existing equipment, the boundary of packet filter function, add the firewall configuration, so that the original router has the function of the firewall. Through a firewall to connect with the required network firewall is no longer needed. Simple protection, it can be directly through the router in the topology structure, boundary routing and firewall joint constitutes the two lines of defense of the computer system, guarantee the safe operation of the computer[19]. The working mechanism is to treat incoming information, exterior routing is mainly responsible for guard against external attacks, only allow external access host defense system, and the inside of the routing to provide the second protection, only accept the host defense of packets, so from a certain extent to ensure the network is always a safe environment. Agent technology work the protocol used to identify users, and can carry on the control to the user's behavior, in the application proxy server, the loop level proxy servers, hosting server and isolation have widely used in the domain name server.

The Network Monitoring and Storage Applications. Condition monitoring of firewall in the network layer can effectively intercept data information, and then through a firewall extracted from various application layer security strategies in the dynamic list, analysis and packet subsequent connection request reasonable and appropriate decisions, each layer of network security detection and control. Timely find security vulnerabilities and malicious attacks, and will these cases recorded, the system administrator according to record screen hole, perfecting the system configuration. Condition monitoring firewall itself according to the records, to better match the corresponding processing method, the real-time dynamic control. In the network layer and top state monitoring, can effective to deal with the layers of loopholes and invasion, makes the dynamic monitoring technology is effectively applied and widely used. Therefore, the application of the dynamic monitoring technology in the safety performance and the interception efficiency are strong. Use a firewall to network information storage function, the firewall can record real-time network at all stages of use, can be used as a firewall for a valid reference network control, easy to analyzing data risks effectively, reduce unnecessary losses.

The Application of the Database Security Maintenance. Important storage area information database as a unit, avoid unit information disclosure is an important function of firewall. Use a firewall to a certain extent, can separate the internal network and external network, legal information are allowed, refused to illegal information access, illegal information quickly to record it to information database, avoid network infected other data, prevent network security problem, at the same time can effectively ensure the sensitive problems not affect local area network globally. Firewall through layer upon layer analysis of the data, not only to be able to effectively filter the information in the database, but also against the database of the network applications to provide the corresponding security services[20]. Firewall in the process of the safe operation of the network system, effectively guarantee the accuracy of the user to find the database information, and can quickly when appear safe hidden trouble to intercept, and promptly provides the user with the effective basis and means of safe and efficient operation of the database. Combined with an efficient database system with the firewall technology can make the firewall technology constantly improve, to ensure the safety of the individuals and enterprises information, to protect the legal interests of individuals and businesses.

\section{Summary}

With the development of computer and communication technology, the computer net will penetrate every field of our social life as an important method of exchanging information. So, recognizing the frangibility and potential threatens of the net and some existent security problems objectively, taking effectively security strategy and ensuring the security of the net information are the things that every country, and group and every person must envisage. From the basic concept and existent problem of the net information security, some digital security technologies, such as information cryptography technology, digital abstract, digital signature, digital envelope and digital 
credentials have been analyzed in the paper. And the application of the firewall technology in the net information security, the functions, types and system structure of the firewall technology, and the development and application of the new generation of the firewall technology have been discussed.

\section{References}

[1] Mohamed G. Gouda, Alex X. Liu. Structured firewall design[J]. Computer Networks. 2006 (4)

[2] B.A. Fessi,M. Hamdi,S. Benabdallah,N. Boudriga. A decisional framework system for computer network intrusion detection[J]. European Journal of Operational Research. 2005 (3)

[3] Katic T, Pale P. Optimization of firewall rules[C]. Proceedings of the ITI 29th International Conf on Information Technology Interfaces, 2007(29): 685-690.

[4] Errin W. Fulp. Optimization of firewall policies using directed acyclic al graphs[C]. Proceedings of the IEEE Internet Management Conference(IM'05), France, 2005.

[5] T. Chomsiri, C. Pornavalai. Firewall Rules Analysis[C]. In Proceedings of International Conference on Security and Management, Las Vegas, USA. 2006(7): 213-219.

[6] Leonardo Maccari,Renato Lo Cigno. Waterwall: a cooperative, distributed firewall for wireless mesh networks[J]. EURASIP Journal on Wireless Communications and Ne . 2013 (1)

[7] Rovniagin, Dmitry,Wool, Avishai. The Geometric Efficient Matching Algorithm for Firewalls[J]. IEEE Transactions on Dependable and Secure Computing. 2011 (1)

[8] S. Pozo,R. Ceballos,R.M. Gasca. Model-Based Development of firewall rule sets: Diagnosing model inconsistencies[J]. Information and Software Technology . 2008 (5)

[9] Mohamed G. Gouda,Alex X. Liu. Structured firewall design[J]. Computer Networks . 2006 (4)

[10] Mikkel Christiansen,Emmanuel Fleury. An MTIDD Based Firewall[J]. Telecommunication Systems . 2004 (2)

[11]T Tran, E Al-Shaer, R Boutaba. PolicyVis: Firewall Security Policy Visualization and Inspection[C]. Conference on Large Installation System Administration Conference, 2007: $1-16$.

[12]Florian Mansmann, Timo Gobel, William Cheswick. Visual analysis of complex firewall configurations[C]. Proceedings of the Ninth International Symposium on Visualization for Cyber Security, New York, USA., 2012(9): 1-8.

[13]Alex X. Liu. Change-Impact Analysis of Firewall Policies[C]. European Conference on Research in Computer Security, 2007: 155-170.

[14]M. Mejri, K. Adi, H. Fujita. Formal Specification and Analysis of Firewalls[C]. Conference on New Trends in Software Methodologies, 2009(199): 284-293.

[15] S. Ioannidis, A.D. Keromytis, S.M. Bellovin, etc. Implementing a distributed firewall[C]. 7th ACM Conference on Computer and Communications Security, 2000: 190-199.

[16]L. Maccari, R. Lo Cigno. Privacy in the pervasive era: A distributed firewall approach[C]. Conference on Wireless On-demand Network Systems and Services, 2012: 23-26.

[17] M. Taghizadeh, A.R. Khakpour, A.X. Liu, S. Biswas. Collaborative firewalling in wireless networks[C]. IEEE International Conference on Computer Communications, 2011, 8(1): 46-50.

[18]R Fantacci, L Maccari, P Neira Ayuso, RM Gasca. Efficient packet filtering in wireless ad hoc networks[C]. IEEE Communications Magazine, 2008, 46(2): 104-110. 
[19]M. Alicherry, A.D. Keromytis, A Stavrou. Evaluating a collaborative defense architecture for MANETs[C]. IEEE international conference on Internet multimedia services architecture and applications, 2009:1-6.

[20]M Alicherry, AD Keromytis. DIPLOMA: Distributed Policy Enforcement Architecture for MANETs[C]. 4th International Conference on Net work and System Security, 2010: 89-98. 